\title{
Micro formed holographic security features in steel
}

\author{
Eckart Uhlmann ${ }^{1,2}$, Stephanie Schulze, née Frenzel ${ }^{1 *}$, Julian Polte ${ }^{2}$, Christoph Hein $^{2}$, Johannes Matthiesen ${ }^{3}$ \\ and Manfred Böge ${ }^{4}$ \\ ${ }^{1}$ Institute for Machine Tools and Factory Management IWF, \\ Technische Universität Berlin, Pascalstr. 8 - 9, 10587 Berlin, Germany \\ ${ }^{2}$ Fraunhofer Institute for Production Systems and Design Technology IPK, Pascalstr. 8 - 9, 10587 Berlin, Germany \\ ${ }^{3}$ HoloDimensions GmbH, In de Butz 4, 22967 Tremsbüttel, Germany \\ ${ }^{4}$ gebr. Böge Metallveredelungs GmbH, Kurt-A. -Körber-Chaussee 27 - 31, 21033 Hamburg, Germany
}

\begin{abstract}
Counterfeiting, with a global trade volume of approximately 450 billion euro a year compromises business results of companies in all industrial segments. At worst, product plagiarism causes severe damages to the individual brand reputation due to product defects or liability issues. Therefore, product integration of inseparable and unique security features is essential for a preserving market share in all manufacturing branches. This paper presents a novel approach regarding a replication technology for the manufacturing of holographic security features. Using a microstructured aluminium substrate with holographic properties as a base material, a forming die is manufactured by a combination of physical vapour deposition (PVD) and galvanic coating processes. Furthermore, a process adapted hardness progression between individual layers was created. By the use of this die, the forming of a holographic microstructure into high strength aluminium alloy (3.3547) and spring steel (1.1248) could be demonstrated within a preliminary study.
\end{abstract}

Keywords: micro-forming, stamping, hard-elastic layer

\section{Introduction}

Holographic labeles are accepted security features for large-scale consumer goods in the form of adhesive tags. However, these labels exhibit the disadvantage of easy removal and imitation. Other proven security solutions as data matrix codes as well as RFID labels do not show significant advantages and include eligible costs that can easily exceed the selling price of a product. Evolved and improved security features therefore have to meet requirements at both, counterfeit protection and economic viability. Holographic structures are outstandingly suitable for the use as security features because of the machine readability on the one hand and an opportunity for the customer to perform a visual authenticity check on the other hand. In order to use holographic structures as a security feature, however, unresolved questions still need to be answered. On the one hand, current research and development is targeting the cost-efficient production of unique and function-adapted holographic structures. On the other hand, the development of technologies for the indelible integration of holographic structures into products is an aim of current scientific and technological development [4].

Furthermore, the challenging process chains for the manufacturing of holographic structures by ultraprecision machining or lithographic techniques causes a reliable and cost-efficient technology for the application of holograms on product surfaces. These are needed to increase the acceptance of holograms as security features. Hence, the replication of microstructures and holograms by embossing is a promising approach. The replication of microstructures by embossing is an established manufacturing process in the field of plastics processing. In particular, hot-embossing is established for the moulding of microstructures and nanostructures into plastic substrates. However and due to the process forces and the effect on the wear of the stamping die, the replication of microstructures into metallic substrates has not been accepted. For this reasons, design and evaluation of a process-adapted stamping die are the subject of the investigation presented.

\section{Development of a stamping die with a process-adapted hardness profile}

The general requirements for a process adapted forming die are a microstructured hard material layer on a hard elastic substratum. The main difficulty in manufacturing a mask following this structure persist in the implementation of the microstructures. This is because hard material layers are not accessible to established methods such as lithography or ultraprecision machining.

\footnotetext{
*Corresponding author: schulze@iwf.tu-berlin.de
} 
For this reason, a completely new approach based on the use of a holographic aluminium shim is presented. The holographic aluminium shim was manufactured by HoloDimensions $\mathrm{GmbH}$ by lithography and roll-to-roll replication technology. Starting from this aluminium shim, the layer structure is performed by means of physical vapour deposition process (PVD). As shown in figure 1, the layer systems consists of a titanium nitride $(\mathrm{TiN})$ and a copper $(\mathrm{Cu})$ layer. The titanium nitride layer represents the hard material layer, the surface and also ensures the transmission of the microstructure into the stamping die. The copper layer forms the adhesion promoter for the subsequent chemical and galvanic nickel (Ni) plating processes.

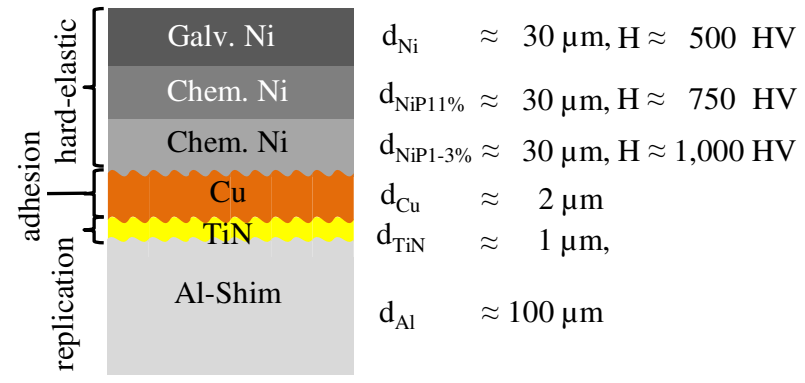

Fig. 1. Layer system consisting of Al-TiN-Cu-NiP-Ni.

In order to implement the previously presented layer system as replication of the holographic aluminium shim, the substrate has to be conditioned for the coating process. This includes a three-stage ultrasonic assisted cleaning process using acetone, isopropanol and deionized water. The deposition of ultra-hard and stoichiometric TiNlayers on the aluminium substrate is performed with an optimised parameter set based on a $\mathrm{Ni}$ target, a sputtering power $\mathrm{P}=600 \mathrm{~W}$, the use of the radio frequency mode (RF) in addition with argon (Ar) and nitrogen $(\mathrm{N})$ process gases. Furthermore, a PVD coating machine tool Z400, LEYBOLD HERAEUS AG, Cologne was used.

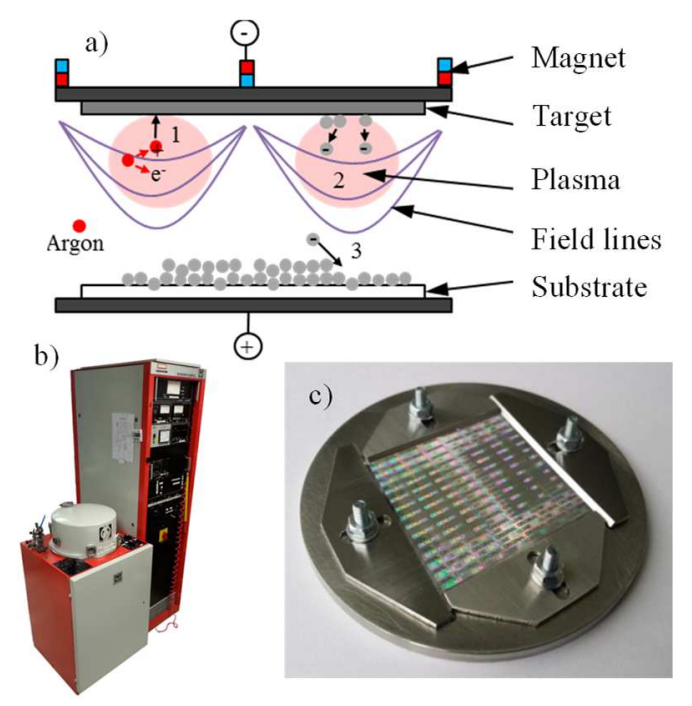

Fig. 2. Schematic representation of the PVD coating process (a), Coating machine tool LH Z400 (b), Substrate holder (c)
In figure 2, the schematic representation of the PVD coating process, the machine tool and the clamping system for the fixture of the aluminium shim are presented.

By the use of optical inspection with a microscope Axio Image 2, CARL ZEISS MICROSCOPY GMBH,

Jena, Germany and a magnification between $10 \mathrm{x} \leq \mathrm{f} \leq 100 \mathrm{x}$, the replication accuracy of the aluminium microstructure in the TiN layer has been assessed (Fig. 3). As a result of the investigation it is shown that the replication of the holographic structure could be done while providing excellent replication quality.

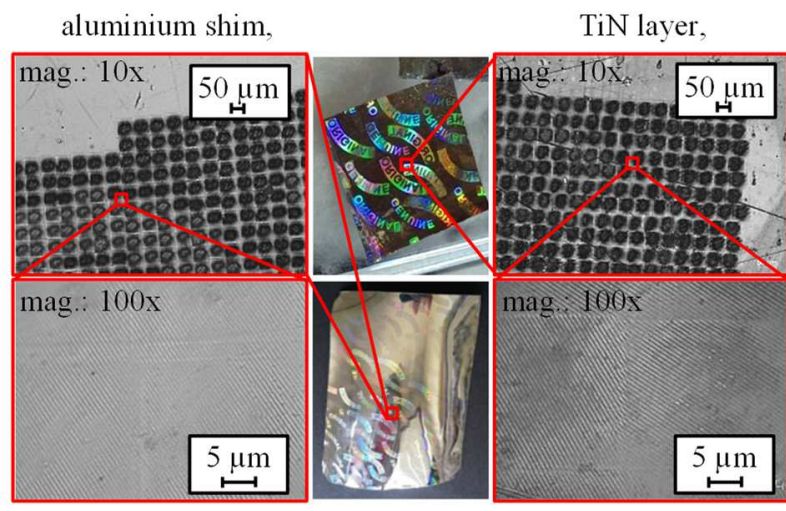

Fig. 3. Comparison of the replication accuracy of hologram structures of the aluminium shim in comparison to the TiN replicate.

Additionally, the replicated holographic structure was evaluated with the method of white-light interferometry and a NewView 5010 ZYGO CORP., Midfield, USA measurement device. The results are shown in Figure 4 exemplarily.
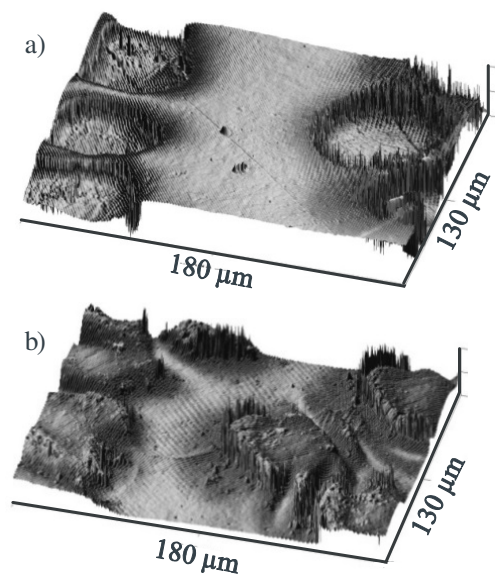

Fig. 4. Comparison of the replication accuracy by means of WLI of a) Al-Shim and b) TiN-replicate.

As shown in figure 4, no significant rounding of sharpedges was detected. During replication by sputtering, the TiN-replicate can be considered as a negative of the holographic aluminium shim. The height peaks occurring in the $3 \mathrm{D}$-profile are artefacts that are based on process- 
related measurement errors and can therefore be neglected. It confirms the already obtained impression that the positive form of the TiN-replicate is the exact negative form of the aluminium shim. Even structures in the nanometer range are transferred in full. Furthermore, the PVD coated $\mathrm{Cu}$-layer is cathodically degreased and etched to enable an adhesion of the nickel phosphorus (NiP) layer. Afterwards the NiP is chemically deposited without an externally applied current. The first NiP-Layer has a phosphorus content of $1 \% \leq \mathrm{n}_{\mathrm{Ph}} \leq 3 \%$ and the second NiP-Layer applied has a phosphorus content of $10.5 \% \leq \mathrm{n}_{\mathrm{Ph}} \leq 12 \%$. The layer composite concludes with an electrolytic nickel plating (pure nickel). Once there is sustainable layer system, the aluminium shim is chemically dissolved.

After all, the stamping die is composed of a base unit (fig. 5) and the holographic stamping foil. The base unit of the stamping die consists of the adapter and the holographic carrier plate. The material used for the composition of the carrier is $\mathrm{X} 153 \mathrm{CrMoV} 12$ steel (1.2379). In order to apply the holographic stamping foil to the carrier plate, cohesive bonding techniques was used.

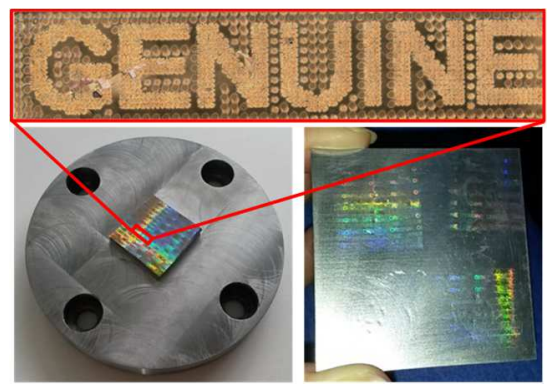

Fig. 5. Embossing result in spring steel strip (1.1248).

For the implementation of the power and path controlled embossing process the materials testing machine tool Z150, ZWICK GMBH, Ulm, Germany shown in figure 6 was used.

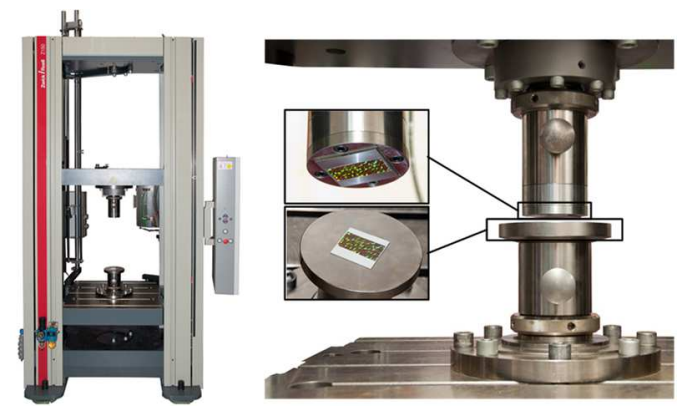

Fig. 6. Stamping test setup on a materials testing machine tool Z150, ZWICK GMBH, Ulm, Germany.

\section{Forming of holographic security features}

For the determination of the forming force $F_{F}$, the following formula can be used [2]:

$$
F_{F}=2 \cdot k_{f o} \cdot s / \eta_{F}
$$

with

$$
k_{f o} \approx 2 \cdot R_{p 0.2} \text {. }
$$

For the forming of high-strength aluminum alloy AMP 8000 with a forming efficiency $\eta_{\mathrm{f}} \approx 0.8$, a die surface $\mathrm{S}=400 \mathrm{~mm}^{2}$, the $0.2 \%$ proof stress of the alloy and a yield strength $\mathrm{R}_{\mathrm{p} 0.2} \approx 120 \mathrm{~N} / \mathrm{mm}^{2}$, a forming force $50 \mathrm{kN} \leq \mathrm{F}_{\mathrm{F}} \leq 150 \mathrm{kN}$ was predefined.

As shown in figure 7 even at low forming forces $\mathrm{F}_{\mathrm{F}}$ a hologram can be identified visually. An increasing forming force $F_{F}$ leads to a reinforced visibility in succession with an improved transmission of the microstructures. An investigation of the processing result by atomic force microscopy (AFM) confirms the result of the visual assessment. The transmission of the majority of the holographic structure is possible even at low forming forces $F_{F}$. However, the holographic effect and the transmission of the height profile increases with an equally rising forming force $\mathrm{F}_{\mathrm{F}}$.
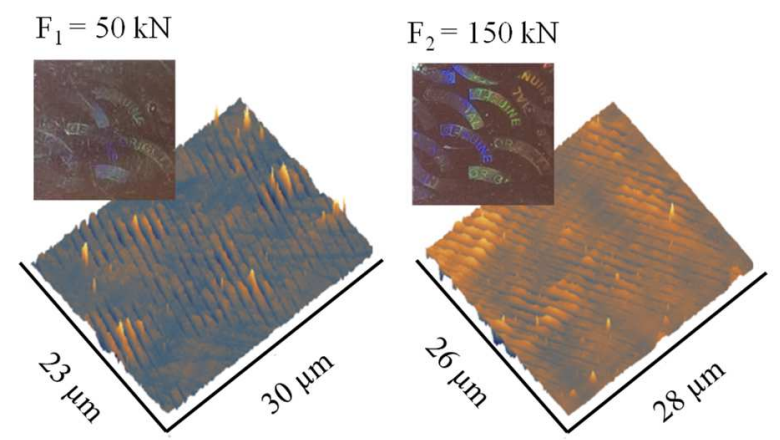

Fig. 7. Comparison

of the embossing results in AMP 8000 with different forming forces $F_{F}$ measured by atomic force microscopy (AFM) and an AFM machine tool N8 NEOS, BRUKER CORP., Billerica, USA.

Nevertheless, the TiN-stamp is subject to signs of wear whose initial stage can be observed in Fig. 8 after embossing no. 50 in the form of chipping in spring steel strip (1.1248).

Beyond that, even with a few forming cycles initial signs of wear are traceable. These show up as breakouts of the TiN-layer and chiping. The breakouts are evidently caused by the elastic movement or compression of the TiN-layer under forming forces $\mathrm{F}_{\mathrm{F}} \leq 150 \mathrm{kN}$. 
The hard-elastic structure of the embossing foil prevents breakage at high forming forces $\mathrm{F}_{\mathrm{F}}$ [3]. As a primary reason for the increased process reliability, the reduction of the compressive stress load by their derivation into the hard-elastic layer structure is presumed $[5,6]$.

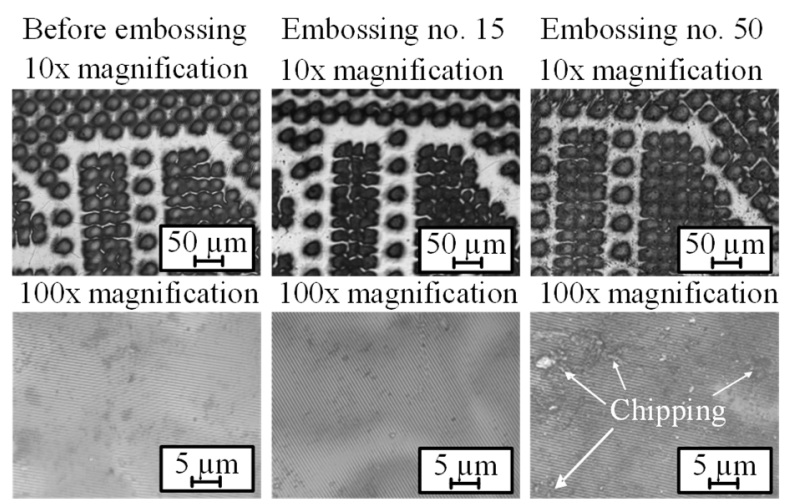

Fig. 8. Comparison of wear behaviour of the holographic TiN die

In addition, WLI-images show that the structural plateau edges of the imaged hologram are reduced in terms of profiling (figure 8), but due to direction of force application the plane surface sections nearly unaffected by wear.

Furthermore, it was discovered that features with an increasingly smaller structural size tend to have a lower wear tilt. Under the assumption of a difference of the surface roughness $\mathrm{Ra}$ between substrate holographic substructures, this fact might preserve the integrity of the holographic structure. The chipping of the ultra-hard TiNlayer, which can be identified as white spots in figures 8 and 9 , is evidently caused by the elastic movement and the compression of the whole forming die under the effect of the forming force $\mathrm{F}_{\mathrm{F}}$. However, the hard-elastic structure of the forming die prevents the breakage of the whole surface at high forming forces [7].

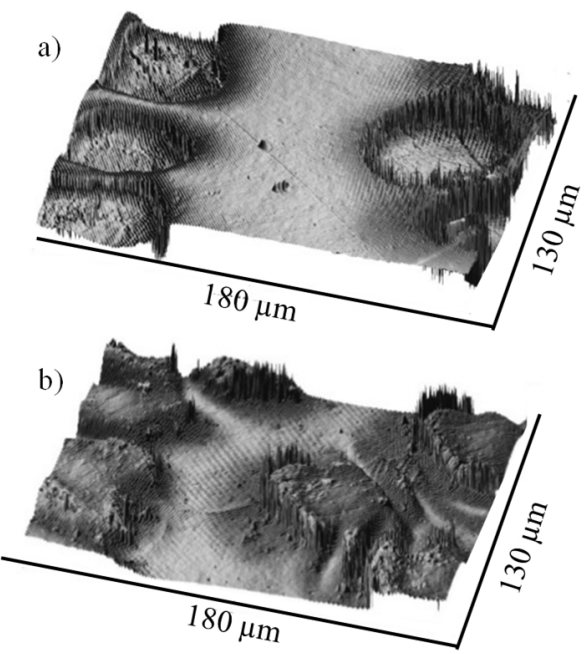

Fig. 9. Wear documentation of the die in new state and after $\mathrm{n}=50$ forming cycles

\section{Outlook}

By the use of the presented method, an enabling technology regarding future forgery-proofing was approved. The replication of holographic structures into the surface of a process adapted forming die was established. By the use of this forming die, a manufacturing process for the forming of holographic structures into materials with a certain market relevance was developed and validated. Based on the forming experiments and with a forming force $\mathrm{F}_{\mathrm{F}} \leq 150 \mathrm{kN}$, a number of cycles $50 \leq \mathrm{n} \leq 500$ can be assumed, depending on the material to be embossed.

The objective of the further studies is to produce a reasonable understanding about the microscopic, structural processes during the forming process and the derivation of measures to minimise wear processes.

\section{References}

1. Abele, E.; Kuske, P.; Lang, H.: Schutz vor Produktpiraterie: Ein Handbuch für den Maschinenund Anlagenbau. Springer, (2011)

2. Kugler, H.: Umformtechnik - Umformen metallischer Konstruktionswerkstoffe. Carl Hanser, München, (2009)

3. Ganzherli, N. M.; Maurer, I. A.; Chernykh, D. F.; Gulyaev, S. N.: Forming the surface relief of complex holographic structures on photosensitive material PFG-01. Journal of Optical Technology Vol. 82, (3), (2015)

4. Vollertsen, F.; Hu, Z.; Schulze Niehoff, H.; Theiler, C.: State of the art in micro forming and investigations into micro deep drawing. Journal of Materials Processing Technology Vol. 151 (3), (2004)

5. Wang, Z.; Shuhui, L.; Wang, X.; Cui, R.; Zhang, W.: Modeling of surface layer and strain hardening effects on micro-bending of non oriented silicon steel sheet. Materials Science and engineering Vol. 711, (2018)

6. Li, Y.; Wang, G.; Liu, S.; Yang, J.; Yang, C.; Zhang, K.: Drawability and size effects for micro-arrayed deep drawing of $\mathrm{Ni}-\mathrm{Co} / \mathrm{GO}$ nanocomposite foils. Journal of Materials Processing Technology Vol. 249, (2017)

7. Obikawa, T.; Hayashi, M.: Ultrasonic-Assisted Incremental Microforming of Thin Shell Pyramids of Metallic Foil. Micromachines Vol. 8(5), (2017) 